\title{
Angiographic and Clinical Assessment of Patients with Cerebral Arterio-Venous Malformations Treated with Microsurgery and Angioembolization
}

\author{
Zahra Janamiri, Yasaman Arjmand, Pooya Torkian* \\ Department of Radiology, Shohada Tajrish Hospital, Shahid Beheshti University of Medical Sciences, Tehran, Iran \\ *Corresponding author: pooyaaig@yahoo.com
}

\begin{abstract}
Introuction: Brain Arteriovenous malformations (AVMs) are the leading cause of intracerebral hemorrhage in the young population. We set out to determine angiographic features of AVMs in pre-operative setting as well as angiographic and neurological and clinical assessment of these AVMs after treatment with either microsurgical approach or angio-embolization. Materials and Methods: From February 2011 to January 2016, 25 patients with cerebral AVMs were admitted to Shohada Tajrish Hospital. Patients underwent angiographic evaluation for AVM grading based on Spetzler Martin grading system. Also, patient's neurological status was recorded. Based on Spetzler Martin score and AVM size, patients underwent either microsurgical approach or angioembolization. Post-operatively, early and long-term neurological examination was performed and obliteration rate was assessed by post-operative imaging study. Results: There were 18 male and 7 female patients with mean age of 42.2 years who presented with cerebral hemorrhage followed by isolated new-onset seizure, progressive new focal neurological deficit and severe new-onset headache in decreasing manner. Seizure was significantly higher in patients with unruptured AVMs ( $\mathrm{P}$ value $<0.01)$. Angiographic study based on Spetzler-Martin grading system showed that most of the patients had grade $3(32 \%)$, followed by grade $4(28 \%)$, grade $2(28 \%)$, grade $5(8 \%)$ and grade $1(4 \%)$. Most AVMs were eloquent with superficial drainage and small sized. Based on AVM size, trend for operation type in small and medium sized AVMs was microsurgical approach followed by angio-embolization. On the other hand, most patients with large-sized AVMs underwent angio-embolization followed by microsurgical approach. Early post-operative examination revealed more new neurological deficit in angio-embolization than microsurgery treated patients ( $\mathrm{P}$ value $<0.05$ ). Improvement in neurological status was observed in microsurgery treated cases compared to angio-embolization ( $\mathrm{P}$ value $<0.05$ ). Long-term follow up showed similar promising results in patients treated with microsurgery which was statistically significant ( $\mathrm{P}$ value $<0.05$ ). Post-operative imaging study demonstrates complete obliteration in 12 patients out of 15 patients (80\%) who underwent microsurgical approach. Complete obliteration was observed in 4 cases out of 10 patients $(40 \%)$ who were treated with angio-embolization. (P value $<0.05$ ). Conclusion: Although results of this study showed better results with microsurgical approach, choosing best treatment option for patients suffering from symptomatic AVMs depends on imaging studies, pre-operative grading and individual-based selection.
\end{abstract}

Keywords: arterio-venous malformation, angiography, spetzler-marting grading system, microsurgery, angio-embolization

Cite This Article: Zahra Janamiri, Yasaman Arjmand, and Pooya Torkian, "Angiographic and Clinical Assessment of Patients with Cerebral Arterio-Venous Malformations Treated with Microsurgery and Angioembolization." American Journal of Medical Case Reports, vol. 5, no. 4 (2017): 101-103. doi: 10.12691/ajmcr-5-4-6.

\section{Introduction}

Brain arteriovenous malformations (AVMs) are abnormal connections between arteries and veins leading to arteriovenous shunting with an intervening network of vessels - the so-called nidus. [1] Large autopsy series have shown the incidence of AVMs to be between $0.04 \%$ and $0.52 \%$. [2,3] Brain AVMs are the leading cause of intracerebral hemorrhage in the young population. They are responsible for $3 \%$ of strokes in young adults and $9 \%$ of subarachnoid hemorrhages (SAH). They may also cause headaches, seizures, or focal neurological deficits. [3] Recently, more AVMs are being incidentally discovered with non-invasive imaging techniques such as CT angiography. [4] The treatment of AVMs has increased in complexity with the development of multimodal approaches that involve open microsurgical excision, endovasculartherapy and radiosurgery. [5] Current therapeutic decisions for cerebral AVMs are commonly based on natural history risk estimates weighed against outcome data from invasive intervention. At present, both natural history and treatment risks remain incompletely defined, especially in Iran. Thus many centers suggest that every AVM patient should be 
managed individually and they have not yet been able to suggest universal criteria for the management of AVMs. $[6,7]$ In this study we aim to evaluate the angiographic results of invasive management of cerebral AVMs and associated complications.

\section{Materials and Methods}

From February 2011 to January 2016, 25 patients with cerebral AVMs with mean age of 42.2 years were admitted to Shohada Tajrish Hospital. Advanced age, systemic malignancy, unreliability for follow-up, previous interventional treatment or radiotherapy, kidney failure with glomerular filtration rate less than $15 \mathrm{~mL} / \mathrm{min}$, deep seated and eloquent sited AVMs (inoperable), severe allergy to contrast media and refusal of consent to study participation were our exclusion criteria. Patients were classified according to the 5-point Spetzler-Martin grading system with its 3 elements of size which scored 1 for maximum diameter of $3 \mathrm{~cm}$ (small-sized); 2 for diameter of 3 to $6 \mathrm{~cm}$ (medium-sized) and 3, for diameter of $6 \mathrm{~cm}$ (large-sized), drainage which scored 1 for AVM with any drainage into the internal deep cerebral venous system, and location which scored 1 for AVM in functionally important, so-called eloquent brain areas, such as the sensorimotor, language, and visual cortex; the hypothalamus and thalamus; the internal capsule; the brain stem; the cerebellar peduncles; and the deep cerebellar nuclei. After angiographic classification and merging with Magnetic Resonance Imaging (MRI) study and randomization, patients underwent either microsurgical approach or angio-embolization. Also, post-operative imaging and neurological examination were recorded. Besides, potential post-operative complications such as new neurological deficits, new abnormalities on imaging studies and surgery related complications were recorded.

Written informed consents were obtained from the patients with ethical approval by Ethics Committee of Shohada Tajrish Hospital under the principles of the Helsinki Declaration.

\section{Results}

There were 18 male and 7 female patients with mean age of 42.2 years with male: female ratio of 2.5 . No significant differences were observed in demographic data of patients. Of all patients who underwent imaging and angiographic study, 14 cases presented with brain hemorrhage; 9 with intra-cerebral hemorrhage (ICH) and intra-ventricular hemorrhage (IVH), 2 with subarachnoid hemorrhage (SAH) and 3 with simultaneous $\mathrm{ICH}$ and SAH. Of patients with unruptured AVM, 7 patients presented with isolated new-onset seizure, 3 with isolated progressive new focal neurological deficit and 1 with severe new-onset headache only. Ruptured AVMs were most commonly less than $3 \mathrm{~cm}$, eloquent sited and superficially drained. Notably, seizure manifestation was significantly higher in patients with unruptured AVMs (P value $<0.01$ ). It was also higher in larger sized, superficial drained and eloquent sited AVMs. Most of the AVMs were located in frontal lobe $(24 \%)$ followed by parietal lobe $(20 \%)$, cerebellum $(16 \%)$, temporal lobe $(12 \%)$ and thalamus $(8 \%)$. Also, angiographic study revealed multi-lobar involvement in 5 patients $(20 \%)$. Angiographic study based on Spetzler-Martin grading system showed that most of the patients had grade $3(32 \%)$, followed by grade $4(28 \%)$, grade $2(28 \%)$, grade $5(8 \%)$ and grade 1 (4\%). Of the AVMs in our study, most were eloquently sited, had superficial drainage and were small sized, followed by medium sized then large sized. Twelve patients had small-sized (less than $3 \mathrm{~cm}$ ) AVMs who were managed with microsurgery $(75 \%)$ followed by angio-embolization (25\%). Seven patients had medium sized AVMs (between $3 \mathrm{~cm}$ to $6 \mathrm{~cm}$ ) who underwent microsurgery $(71.4 \%)$ followed by angio-embolization $(28.5 \%)$. On the other hand, of 6 patients with large-sized (more than $6 \mathrm{~cm}$ ) AVMs, angio-embolization approach was considered for 5 cases $(83.3 \%)$, followed by microsurgical approach for 1 patient (16.6\%). Early post-operative neurological examination revealed new neurological deficit in 6 patients treated with angio-embolization followed by 2 patients treated with microsurgery ( $\mathrm{P}$ value $<0.05$ ). Although, 8 cases who underwent microsurgery showed improved neurological examination, which same result was observed in 1 patient who was treated with angio-embolization ( $\mathrm{P}$ value $<0.05)$. However, 6 patients who were treated with microsurgery and 2 patients who underwent angio-embolization, did not manifest any improvement or deterioration in their neurological examination compared to our initial recordings. Long term post-operative neurological examination showed partial improvement in 11 cases of microsurgery-treated patients and in 3 cases of angio-embolization ( $\mathrm{P}$ value $<0.05$ ). Complete resolution of initial deficit was observed in 4 patients who were treated with microsurgery as well as in 1 patient who was treated with angio-embolization. However, 5 patients who were treated with microsurgery and 1 patient who underwent angio-embolization did not show any changes from their initial recorded neurological examination. Besides, no death was observed in mentioned period of our study. Moreover, post-operative imaging study demonstrates complete obliteration in 12 patients out of 15 patients $(80 \%)$ who underwent microsurgical approach. Complete obliteration was observed in 4 cases out of 10 patients $(40 \%)$ who were treated with angio-embolization. ( $\mathrm{P}$ value $<0.05)$.

\section{Discussion}

Intracranial hemorrhage is the most common manifestation of an AVM and accounts for as many as $50 \%$ of initial presentations, resulting in a first-ever hemorrhage rate of 0.55 per 100,000 person years. [8] The annual hemorrhage risk may be as low as $0.9 \%$ or as high as $34 \%$ in patients depending on the AVMs characteristics and history of previous rupture. [9] Natural history studies of untreated AVMs have demonstrated an overall $2 \%$ to $4 \%$ annual rate of hemorrhage, with a combined annual morbidity and mortality of approximately 3\%. [10] Although the exact number of asymptomatic patients is unknown, clinical studies report that $2 \%$ to $4 \%$ of AVMs detected are incidental findings. [11] Care focuses on 
prevention of hemorrhage, seizures, and neurological deficits associated with AVMs. According to patient selection, ruptured AVMs had significantly been managed more with non observational modalities. Size and Spetzler-Martin grade have had a significant effect on patient selection in our center and the patients with lower grades and smaller sizes have been significantly considered more for interventional management. [12] Transient complications were seen in $20 \%$ of the grade 3 or more AVMs, compared to $0 \%$ in fewer grades. [13] Permanent complications were however equal in both groups. The data is confirmative of other studies that have also noted higher rates of complications in larger sized AVM's. [14] Transient complications were most common in ruptured AVMs whereas permanent complications were most common in the unruptured group. Outcomes and complications were shown to be impacted significantly by age and hemorrhage, which is consistent with other studies that older patient age and non-hemorrhagic presentation predict new complications. [15] The incidence of hemorrhage was slightly lower than related studies, where it had been reported above 50\%. [16] In two population based studies, $38 \%$ and $65 \%$ of patients with AVM had hemorrhage, with a peak incidence in the fifth decade of age. Although hemorrhage from an AVM can be disabling or deadly, the course in many non-operated high-grade AVMs (Spetzler Martin grades 4 and 5) can be quite benign, if compared with their intervention risk. This may justify conservative treatment in high-grade/high-risk AVM s. [17,18] As each of these procedures has its own associated complications and limitations, choosing the modality of choice for treatment presents a significant dilemma for every physician. In addition, the numerous variables affecting the results and uncertainties surrounding the natural history have also made treatment selection more difficult. [19] Advanced technology and improved understanding of the natural history of AVMs, has led to the reconsideration of the management of these lesions, which was once only comprised of microsurgical removal.

\section{Conclusion}

Among all modalities of treatment, microsurgical removal still remains the definitive form of treatment for low grade AVMs. On the other hand, management of large and deep AVMs (thalamus, brain stem, and basal ganglia) remains challenging and is still associated with relatively high morbidity; especially in large deep or Spetzler-Martin grade 4 and 5 AVMs.

The final decision to treat patients diagnosed with cerebral AVMs is recommended to be based on comparing the risks of observation against treatments directed at eliminating the AVM; this is by considering the patients AVM characteristics along with their age, job, economic situation and the centers local expertise and facilities.

\section{References}

[1] Kim, H., Su, H., Weinsheimer, S., Pawlikowska, L., Young, W.L., "Brain arteriovenous malformation pathogenesis: a response-toinjury paradigm," Acta Neurochir Suppl, 111:83-92. 2011.

[2] Al-Shahi, R., Fang, J.S., Lewis, S.C., Warlow, C.P., "Prevalence of adults with brain arteriovenous malformations: a community based study in Scotland using capture-recapture analysis," Journal of Neurology, Neurosurgery \& Psychiatry, 73(5). 547-51. 2002.

[3] Guo, P., Zhao, J.Z., "Clinical characteristics and surgical outcomes of 3094 patients with cerebral arteriovenous malformation," Zhonghua yi xue za zhi, 91(39). 2740-3. 2011.

[4] Gabriel, R.A., Kim, H., Sidney, S., McCulloch, C.E., Singh, V., Johnston, S.C., Ko, N.U., Achrol, A.S., Zaroff, J.G., Young, W.L., "Ten-year detection rate of brain arteriovenous malformations in a large, multiethnic, defined population," Stroke, 41(1). 21-6. 2010.

[5] van Beijnum, J., van der Worp, H.B., Buis, D.R., Salman, R.A., Kappelle, L.J., Rinkel, G.J., van der Sprenkel, J.W., Vandertop, W.P., Algra, A., Klijn, C.J., "Treatment of brain arteriovenous malformations: a systematic review and meta-analysis," Jama, 306(18). 2011-19. 2011.

[6] Ogilvy, C.S., Stieg, P.E., Awad, I., Brown, R.D., Kondziolka, D., Rosenwasser, R., Young, W.L., Hademenos, G., "Recommendations for the management of intracranial arteriovenous malformations," Stroke, 32(6). 1458-71. 2001.

[7] Fine, A.D., Beauregard, C.L., Day, A.L., "20 Arteriovenous Malformations of the Cerebellar Vermis and Hemispheres," Intracranial Arteriovenous Malformations, 285. 2006.

[8] Berman, M.F., Sciacca, R.R., Pile-Spellman, J., Stapf, C., Connolly Jr, E.S., Mohr, J.P., Young, W.L., "The epidemiology of brain arteriovenous malformations," Neurosurgery, 47(2). 389-97. 2000.

[9] Kim, H., Pawlikowska, L., Weinsheimer, S., Kwok, P.Y., Zaroff, J.G., McCulloch, C.E., Young, W.L., "Genome-wide association study of intracranial hemorrhage in brain arteriovenous malformation (BAVM) patients," Stroke, 41(e11). P37. 2010.

[10] Ondra, S.L., Troupp, H., George, E.D., Schwab, K., “The natural history of symptomatic arteriovenous malformations of the brain: a 24-year follow-up assessment," Journal of neurosurgery, 73(3). 387-91. 1990.

[11] Davies, J.M., Yanamadala, V., Lawton, M.T., "Comparative effectiveness of treatments for cerebral arteriovenous malformations: trends in nationwide outcomes from 2000 to 2009," Neurosurgical focus, 33(1). E11. 2012.

[12] Söderman, M., Andersson, T., Karlsson, B., Wallace, M.C., Edner, G., "Management of patients with brain arteriovenous malformations," European journal of radiology, 46(3). 195-205. 2003.

[13] Pandey, P., Marks, M.P., Harraher, C.D., Westbroek, E.M., Chang, S.D., Do, H.M., Levy, R.P., Dodd, R.L., Steinberg, G.K., "Multimodality management of Spetzler-Martin Grade III arteriovenous malformations: Clinical article," Journal of neurosurgery, 116(6). 1279-88. 2012.

[14] Wedderburn, C.J., van Beijnum, J., Bhattacharya, J.J., Counsell, C.E., Papanastassiou, V., Ritchie, V., Roberts, R.C., Sellar, R.J., Warlow, C.P., Salman, R.A., "Outcome after interventional or conservative management of unruptured brain arteriovenous malformations: a prospective, population-based cohort study," The Lancet Neurology, 7(3). 223-30. 2008.

[15] Brown Jr, R.D., Wiebers, D.O., Torner, J.C., O'Fallon, W.M., "Frequency of intracranial hemorrhage as a presenting symptom and subtype analysis: a population-based study of intracranial vascular malformations in Olmsted County, Minnesota," Journal of neurosurgery, 85(1). 29-32. 1996

[16] Van Beijnum, J., Bhattacharya, J.J., Counsell, C.E., Papanastassiou, V., Ritchie, V., Roberts, R.C., Sellar, R.J., Warlow, C., Salman, R.A., "Patterns of Brain Arteriovenous Malformation Treatment," Stroke, 39(12). 3216-21. 2008.

[17] Muñoz, F., Clavel, P., Molet, J., Castaño, C., De Teresa, S., Solivera, J., De Quintana, C., Tresserras, P., Rodríguez, R., Bartumeus, F., "Current management of arteriovenous malformations. Retrospective study of 31 cases and literature review," Neurocirugia (Asturias, Spain), 18(5). 394-404. 2007.

[18] Altay, T., "Management of Arteriovenous Malformations Related to Spetzler-Martin Grading System," Journal of Neurological Surgery Part A: Central European Neurosurgery, 73(05). 307-19. 2012.

[19] Gross, B.A., Du, R., "Surgical and radiosurgical results of the treatment of cerebral arteriovenous malformations," Journal of Clinical Neuroscience, 19(7). 1001-4. 2012.

[20] Nikouei, A., Seddighi, A., Seddighi, A.S., "The Results of Image Guided Surgery Using Neuronavigation in Resection of Cerebral Gliomas in Eloquent Cortical Areas," Archives of Physical Medicine and Rehabilitation, 97(10). e69-70. 2016.

[21] Yang, S.Y., Kim, D.G., Chung, H.T., Paek, S.H., "Radiosurgery for unruptured cerebral arteriovenous malformations Long-term seizure outcome," Neurology, 78(17). 1292-8. 2012. 